CLINICAL STUDY

\title{
Functional and total IGFBP 3 for the assessment of disorders of the GH/IGF 1 axis in children with chronic kidney disease, GH deficiency, or short stature after SGA status at birth
}

\author{
A K Büscher, R Büscher, L Pridzun ${ }^{2}$, M Langkamp ${ }^{2}$, N Wachendorfer ${ }^{2}$, P F Hoyer, M B Ranke ${ }^{1}$ and B P Hauffa \\ Department of Pediatrics II, Pediatric Nephrology, Endocrinology and Diabetology, Gastroenterology and Transplant Medicine, University Children's \\ Hospital, University of Duisburg-Essen, Hufelandstraße 55, D-45122 Essen, Germany, ${ }^{1}$ Pediatric Endocrinology and Diabetology, University Children's \\ Hospital, University of Tübingen, 72076 Tübingen, Germany and ${ }^{2}$ Mediagnost Corporation, 72770 Reutlingen, Germany \\ (Correspondence should be addressed to B P Hauffa; Email: berthold.hauffa@uk-essen.de)
}

\begin{abstract}
Objective: IGFBP3 immunoreactivity may appear elevated in patients with chronic kidney disease (CKD), in part due to accumulation of low molecular fragments. The importance of these IGFBP3 variants for binding and inactivation of IGF1 and their relevance for the impaired growth of uremic children are unclear. Nevertheless, IGFBP3, measured as total (t-)IGFBP3, is frequently used as a diagnostic parameter in pediatric CKD patients. A new assay for functional (f-)IGFBP3 exclusively detects IGFBP3 capable of IGF binding. The aim of the study was to evaluate the significance of f-IGFBP3 measurements for the assessment of uremic abnormalities of the GH/IGF1 axis.

Design: Prospective cross-sectional study.

Methods: t-IGFBP3, f-IGFBP3, and IGF1 were measured in pediatric CKD patients, including patients with CKD stage 3-4 not on dialysis (CKD, $n=33$ ), on dialysis treatment (DT, $n=26$ ), patients after renal transplantation (RTx, $n=89$ ), healthy children $(n=29)$, children with GH deficiency (GHD, $n=42$ ), and small for gestational age (SGA) children (SGA, $n=34$ ).

Results: Mean t-IGFBP3 SDS was elevated in CKD, DT, and RTx children compared with controls and GHD patients $(P \leq 0.0004)$. Highest values were reached in DT $(P<0.0001$ vs all groups $)$. In contrast, mean f-IGFBP3 was similar in all groups $(P=0.30)$.

Conclusions: Pediatric CKD patients displayed elevated serum concentrations of t-IGFBP3 but not f-IGFBP3, supporting the hypothesis that IGFBP3 fragments not binding IGF1 accumulate during uremia. f-IGFBP3 is an indicator of IGFBP3 fragmentation and seems to reflect IGF1 binding in CKD better than t-IGFBP3. However, the role of f-IGFBP3 for the diagnosis of disturbances of the GH/IGF hormonal axis appears to be limited.
\end{abstract}

European Journal of Endocrinology 166 923-931

\section{Introduction}

Children with chronic kidney disease (CKD) often suffer from severe growth retardation $(1,2)$. Not only malnutrition, metabolic acidosis, chronic inflammation, renal osteodystrophy but also disturbances of the somatotropic hormone axis contribute to this state $(2,3,4)$. In uremic children, growth can be improved significantly by treatment with recombinant $\mathrm{GH}(5,6)$. Following renal transplantation (RTx), with normalization of renal function and improvement of the hormonal disturbances, catch-up growth is noted in most of the children. Sixty-eight percent of the transplanted children reach a normal adult height (7).

Biochemically, longitudinal growth and bone mass are regulated by $\mathrm{GH}$, whose effects are predominantly mediated by IGF1. The bioavailability and functionality of IGF1 are regulated by IGF-binding proteins (IGFBPs)
$(8,9)$. Seventy-five to eighty percent of IGF1 in the circulation is bound in a ternary $150 \mathrm{kDa}$ complex, consisting of IGFBP 3 or -5 , IGF1, and the acid-labile subunit $(10,11,12)$. IGF1 binding by IGFBPs is thought to inhibit interaction with the IGF receptor, thereby weakening the signal that promotes longitudinal growth (12). An important mechanism to release IGF1 from its ternary complex is the limited proteolysis of IGFBP by specific proteases. The emerging fragments exhibit lower affinity for IGF1 with subsequent dissociation of IGF1, resulting in an increased IGF1 bioactivity (13).

During uremia, IGF1 concentrations and, even more so, IGFBP3 immunoreactivity are increased within or exceeding the upper limit of the normal range $(14,15,16)$. In the light of reduced IGF1 production rates, evidenced by reduced mRNA levels in liver tissue (17), and reduced IGF bioactivity, the normal 
immunoreactive IGF1 concentrations seen in uremia are thought to result from decreased IGF degradation caused by enhanced IGFBP binding $(14,18)$. Binding to IGFBP protects IGF1 from metabolic degradation but could, at the same time, inhibit IGF1 interaction with its receptor, resulting in an impaired IGF1 bioactivity $(8,19)$.

Increased IGFBP3 immunoreactivity under uremic conditions is not exclusively caused by intact molecules. Significant immunoreactivity appears to result from accumulation of IGFBP 3 fragments of 19 and $26 \mathrm{kDa}$ size due to a reduced clearance $(15,16)$.

Although alterations of the GH/IGF axis under uremic conditions are complex and often difficult to interpret, IGF1 and IGFBP 3 are frequently measured in patients with CKD (20). In general, measurements of IGFBP3 are performed using commercially available assays quantifying total IGFBP3 immunoreactivity (t-IGFBP3). The results are used, in combination with the height velocity, to initiate or adjust GH therapy. As the immunoreactivity of t-IGFBP 3 in CKD does also include inactive fragments, measurement of t-IGFBP3 by routine immunoassay methods may not reflect the effective hormonal status of these patients.

Recently, an immunoassay for IGFBP 3 has been described that exclusively measures the IGF1-binding molecular variants of IGFBP3 (functional (f-)IGFBP3) (21). We tested this immunoassay in a cohort of children with different stages of CKD and after RTx. Data were compared with those from a control group of healthy children and two other groups of patients with disordered growth (GH deficiency (GHD) and short stature due to small for gestational age (SGA) status at birth) but normal renal function. We also wanted to determine how f-IGFBP3 and related parameters vary with age in healthy children. The aim of this study was to assess the outcome of f-IGFBP3 measurements as an alternative to t-IGFBP3 in the clinical setting of disturbances of the GH/IGF1 axis in renal insufficiency.

\section{Patients and methods}

\section{Patients}

We prospectively analyzed data of 224 pediatric patients with CKD, GHD, or short stature after SGA status at birth and 29 healthy children who served as a control group. The latter children were inpatients of the
University Children's Hospital or the ENT Department of the University Duisburg-Essen admitted for planned minor surgery or minor diseases that did not impair renal function or influence the metabolic or endocrine status. The control children had no history of chronic diseases and did not receive any regular medication. Their height was within the \pm 2 s.D. range for chronological age (CA).

The patients' cohort consisted of 33 patients with CKD not treated with dialysis (CKD group; CKD stage 3-4), 26 patients on dialysis treatment (DT group; nine patients on hemodialysis and 17 patients on peritoneal dialysis), and 89 patients after kidney transplantation (RTx group). Etiology of renal failure was obstructive uropathy $(n=15)$, congenital anomalies of the kidney and the urinary tract $(n=27)$, hemolytic uremic syndrome $(n=18)$, nephrotic syndrome $(n=21)$, nephronophthisis $(n=17)$, autoimmune kidney disease $(n=13)$, syndromal kidney disease $(n=14)$, autosomal recessive polycystic kidney disease $(n=6)$, neurogenic uropathy $(n=2)$, hyperoxaluria type I $(n=4)$, and other renal disorders $(n=11)$. Forty-two non-CKD patients were diagnosed to have GHD group with a maximum $\mathrm{GH}$ level below $10 \mu \mathrm{g} / \mathrm{l}$ in at least two tests. Thirty-four patients fulfilled the auxological criteria for $\mathrm{GH}$ therapy due to SGA status at birth and subsequent impaired growth (SGA group). No GFR data were available for the GHD and the SGA group. The patient groups and the control group differed with regard to their mean age and GFR (Table 1). None of the patients received GH therapy. The concomitant medication of the patients with CKD included, if necessary, bicarbonate, antihypertensive drugs, erythropoietin, oral iron, and phosphate binders. The stages of CKD were defined using the KDOQI criteria of the National Kidney Foundation (22): CKD stage 3: GFR $30-60 \mathrm{ml} / \mathrm{min}$ per $1.73 \mathrm{~m}^{2}$; CKD stage $4:$ GFR $15-30 \mathrm{ml} / \mathrm{min}$ per $1.73 \mathrm{~m}^{2}$. Patients on dialysis started DT at a GFR of $\leq 10-15 \mathrm{ml} / \mathrm{min}$ per $1.73 \mathrm{~m}^{2}$. Other inclusion criteria were age $<20$ years and written informed consent by the parents or legal guardians.

\section{Laboratory methods}

Blood samples were collected in the morning after overnight fasting. In infants, duration of fasting was at least $4 \mathrm{~h}$. Serum was immediately frozen at $-80^{\circ} \mathrm{C}$ until analyzed. Samples were analyzed for t-IGFBP 3 and f-IGFBP3 using commercially available assays (IGFBP3

Table 1 Characteristics of healthy children (controls) and patients groups.

\begin{tabular}{|c|c|c|c|c|c|c|c|}
\hline & Controls & CKD & DT & RTx & GHD & SGA & $\boldsymbol{P}$ \\
\hline Subject number & 29 & 33 & 26 & 89 & 42 & 34 & - \\
\hline Female/male ratio (no. of females) & $0.93(14)$ & $0.74(14)$ & $0.53(9)$ & $0.65(35)$ & $0.45(13)$ & $0.89(16)$ & 0.64 \\
\hline Age (years) (mean \pm s.D.) & $10.03 \pm 4.03$ & $10.92 \pm 5.79$ & $11.50 \pm 5.50$ & $12.98 \pm 4.66$ & $6.89 \pm 2.73$ & $6.95 \pm 2.66$ & $<0.0001$ \\
\hline GFR & $107.8 \pm 20.5$ & $22.1 \pm 13.3$ & $13.92 \pm 6.9$ & $69.1 \pm 19.6$ & - & - & $<0.0001$ \\
\hline
\end{tabular}

$P$ values (ANOVA) indicate differences between groups. 
ELISA and Functional IGFBP3 LIA; Mediagnost, Reutlingen, Germany). The functional assay (ligand immunofunctional assay, LIA) quantifies only those IGFBP3 molecules capable of binding labeled IGF1, called functional molecules. The LIA is designed as a sandwich assay, using an immobilized IGFBP3 polyclonal antibody as solid phase and biotinylated IGF1 as the capture molecule. The sample is first treated with a dilution buffer that releases all naturally bound IGF1s from their binding proteins. Aliquots of this solution are then added to microtiter plate wells containing excess soluble biotinylated IGF1. The walls of the wells are coated with IGFBP 3 antibody. The biotinylated IGF1 occupies all existing specific binding sites of the serum IGFBP 3 molecules. Further, IGFBP3 binds to the IGFBP 3 antibody. Although all IGFBP 3 molecules are bound to the microtiter plate, only those complexed with biotinylated IGF1 are involved in signal generation. Thus, IGFBP3 fragments that do not bind IGF1 are not detected.

Both assays are calibrated against recombinant hIGFBP3. When dilutions $(6 \mu \mathrm{g} / \mathrm{l})$ of two different recombinant hIGFBP3 preparations (R\&D Systems, Minneapolis, MN, USA, and Novozymes GroPrep, Adelaide, Australia) in assay buffer were measured using the f-IGFBP3 assay, recovery was 98.6 and $93.6 \%$. Serial dilutions with assay buffer (1:50 to $1: 300$ ) of two patient samples (mean f-IGFBP3 concentration 1.78 and $2.36 \mathrm{ng} / \mathrm{ml}$ ) revealed coefficients of variation $(\mathrm{CV})$ of 4.4 and $2.5 \%$ respectively. Further details regarding validation and technical properties of the f-IGFBP 3 assay (LIA) have been published elsewhere (21). The interassay CV was $6.8 \%$ for f-IGFBP 3 and $6.3 \%$ for t-IGFBP 3 , the intra-assay variability was $5.6 \%$ for f-IGFBP 3 and $4.51 \%$ for t-IGFBP3. The crossreactivity of both assays with IGFBP1 and -2 $(1000 \mu \mathrm{g} / \mathrm{l})$, or with IGFBP4 and $-5(200 \mu \mathrm{g} / \mathrm{l})$, was $<0.03 \%$. Measurement of both $\mathrm{f}-$ and t-IGFBP 3 was not influenced by serum triglyceride concentrations up to $100 \mathrm{~g} / \mathrm{l}(21)$.

IGF1 was measured using an IGFBP-blocked commercial ELISA (IGF1 ELISA, Mediagnost; calibrated against WHO NIBSC 02/254; interassay CV 7.4\%, intra-assay variability $4.8 \%$ ). In this assay system, interference of IGFBPs with IGF1 detection is diminished by adding excess IGF2.

For IGF1 and t-IGFBP3, the original data were transformed into SDS using the reference data of Blum \& Breier (23). We also calculated the IGF1/IGFBP3 molar ratio, which in cancer epidemiological studies had been used as a surrogate parameter associated with IGF1 bioactivity (24). The f-/t-IGFBP3 molar ratio was calculated to serve as an index of IGFBP 3 fragmentation. A ratio close to 0 would indicate almost complete fragmentation compatible with loss of function whereas a ratio close to 1 would indicate the presence of mostly intact IGFBP3 molecular variants.

\section{Measurement of other analytes}

Creatinine was determined colorimetrically by the Jaffe reaction. The creatinine clearance was calculated according to the Schwartz formula (25).

\section{Ethics approval}

The study protocol was approved by the ethics committee of the University Duisburg-Essen (registry no. 04-2393). Patients and controls were entered into the study after having obtained written informed consent from the parents and, if appropriate, also from the patients.

\section{Statistical analysis}

Results are given as arithmetic mean \pm 1 s.D. (mean \pm s.D.) or as least squares mean with $95 \%$ confidence interval (CI), as indicated. The level of statistical significance was defined as $P<0.05$. Diagnostic groups were tested for sex differences using the $\chi^{2}$ test. Continuous variables were compared with ANOVA or ANCOVA (Proc GLM), correcting for multiple comparisons by Tukey's test. The model used the patient diagnostic groups as independent variable. GFR, IGF1 SDS, f-IGFBP3, t-IGFBP3, t-IGFBP3 SDS, IGF1/t-IGFBP3 molar ratio, IGF1/f-IGFBP3 molar ratio, and the f-IGFBP3/t-IGFBP3 ratio were included as dependent variables. CA was included as a covariable. Data were also analyzed for possible interactions of diagnostic groups with CA. Correlations were performed by linear regression using Spearman's correlation coefficient. Statistical analysis was carried out using the SAS software (SAS Corporation, Cary, NJ, USA).

\section{Results}

\section{Comparison between groups}

As diagnostic groups and controls differed with regard to age $(P<0.0001$; Table 1$)$, data were analyzed using age as a covariate. With this, differences between groups could be demonstrated for all hormonal variables and ratios (IGF1 SDS, t-IGFBP3, t-IGFBP3 SDS, IGF1/f-IGFBP3 ratio, IGF1/t-IGFBP3 ratio, and f-IGFBP3/t-IGFBP3 ratio; $P \leq 0.001$ ), except for f-IGFBP3. Hormonal variables and their ratios according to CA for normal children, patients with CKD stage 3-4 (CKD), children undergoing DT, patients with GHD, and patients born SGA are depicted in Fig. 1.

For f-IGFBP3 serum concentration, ANCOVA did not reveal any differences between healthy children, children with CKD, under DT or after RTx, or children with GHD or SGA $(P=0.30$; Fig. 2 , panel A). This was in contrast to t-IGFBP3, where serum concentrations in the CKD, DT, and RTx groups were significantly elevated over controls and the GHD group $(P \leq 0.0004)$. 


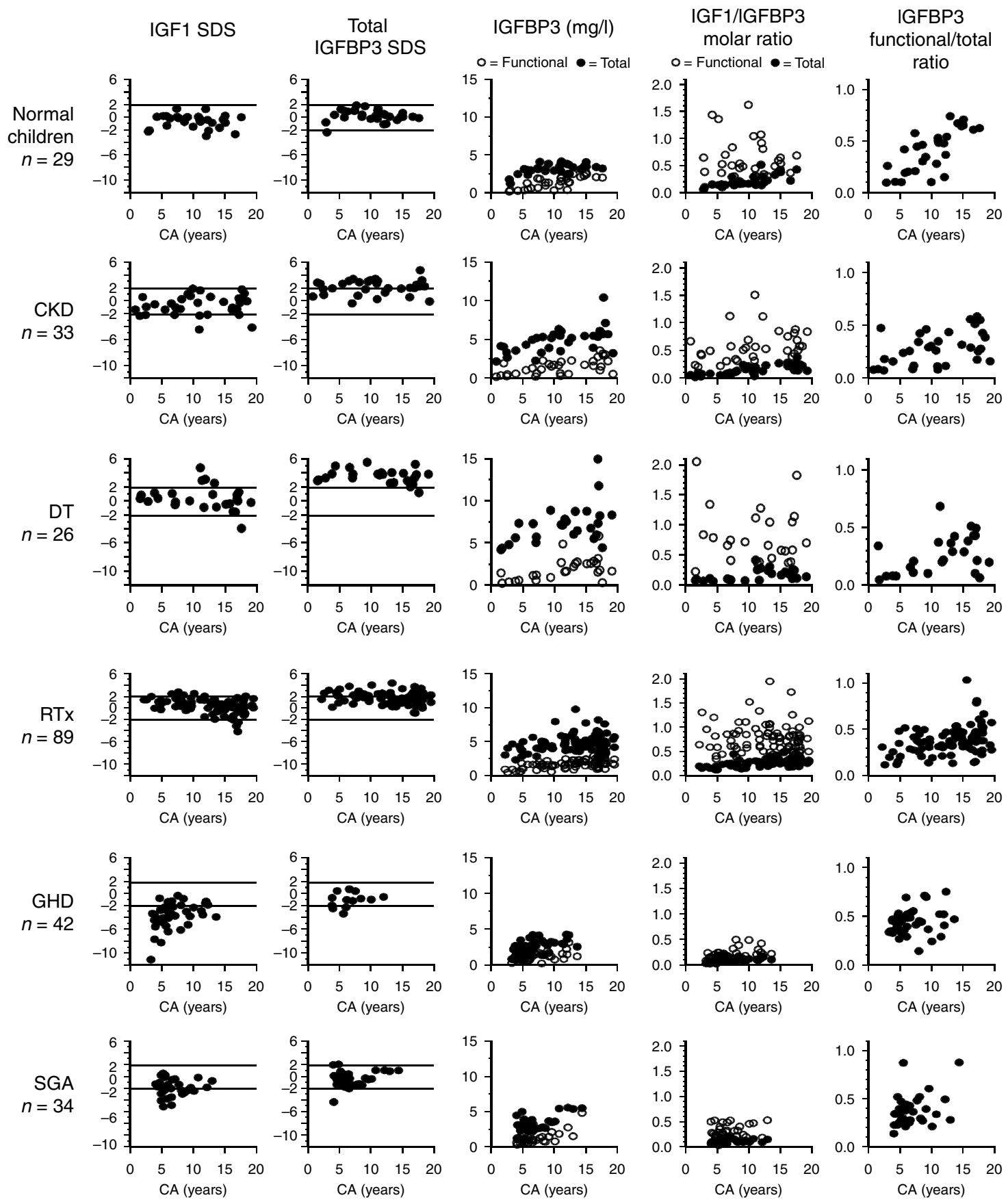

Figure 1 IGF1 SDS and total (t)-IGFBP3 SDS, functional (f) (open circle) and total (t) (closed circle) IGFBP3 serum concentrations, IGF1/f-IGFBP3 (open circle) and IGF1/t-IGFBP3 (closed circle) molar ratios, and f-IGFBP3/t-IGFBP3 ratio according to chronological age (CA) for normal children, patients with chronic kidney disease stage 3-4 (CKD), children undergoing dialysis treatment (DT), patients with GH deficiency (GHD), and patients born small for gestational age (SGA).

t-IGFBP3 concentration was highest in the DT group, where it was elevated even over the RTx group $(P<0.0001$; Fig. 2, panel B). No interaction between age and diagnostic group was found for any of these two parameters.
The IGF1/IGFBP3 molar ratio has been viewed by some authors as a measure for inhibition of IGF1 action, with a low ratio due to excess IGFBP3 reflecting increased IGF sequestration and low IGF bioactivity (reviewed by $(26,27,28))$. When this ratio is calculated 

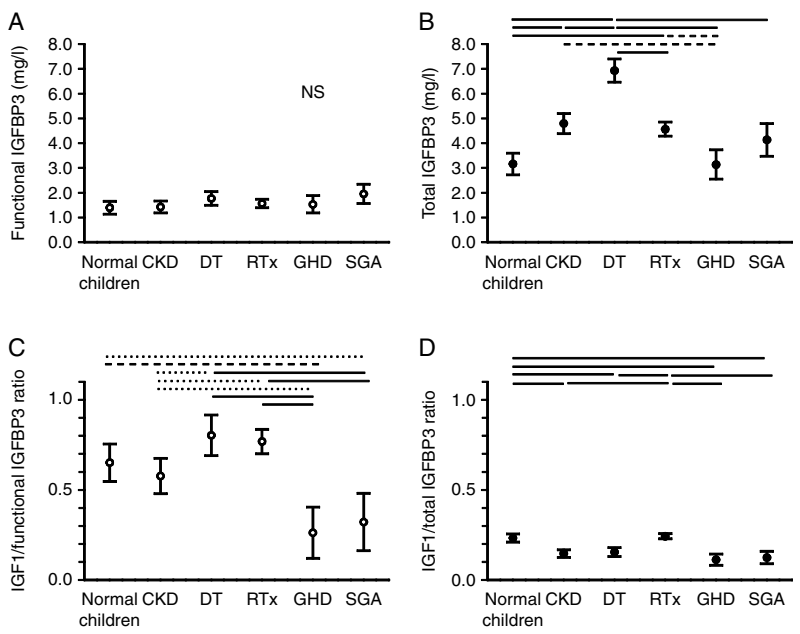

Figure 2 Functional (f)-IGFBP3 serum concentrations (panel A), total (t)-IGFBP3 serum concentrations (panel B), IGF1/f-IGFBP3 molar ratio (panel C), and IGF1/t-IGFBP3 molar ratio (panel D) according to the diagnostic group for normal children, children with chronic kidney disease stage 3-4 (CKD), children undergoing dialysis treatment (DT), children after renal transplantation (RTx), children with GH deficiency (GHD), and children born small for gestational age (SGA) (ANCOVA; covariate chronological age). Circles represent least square means, error bars indicate the $95 \%$ $\mathrm{Cl}$. Horizontal lines indicate differences between groups, with $P<0.05$ (dotted line), $P<0.001$ (dashed line), or $P<0.0001$ (solid line); NS, not significant.

based on t-IGFBP3, the upper limit of the 95\% CI does not exceed 0.23 in all groups (Fig. 2, panel D). The IGF1/t-IGFBP3 ratio is highest in the controls, exceeding that of the DT, GHD, and SGA children $(P<0.0001)$. CKD and DT children have lower ratios than the RTx children and higher ratios than the GHD children. RTx children have a higher ratio than those with GHD and SGA $(P<0.0001)$. Also, there is a significant influence of an interaction between age and diagnostic group on the IGF1/t-IGFBP3 ratio $(P=0.0004)$.

In contrast, IGF1/IGFBP3 molar ratio based on f-IGFBP3 concentrations is generally higher, with the lower and upper limits of the 95\% CI for the groups spanning a range between 0.12 and 0.92 (Fig. 2, panel C). The IGF1/f-IGFBP 3 ratio is lowest for the GHD and SGA children, being lower than that for the DT and RTx children $(P<0.0001)$ and the controls $(P<0.01)$. GHD children also have a lower ratio than CKD children $(P<0.01)$, whereas DT and RTx children have a higher ratio than CKD children $(P<0.05)$. There is no influence of an interaction between age and diagnostic group on the IGF1/f-IGFBP 3 ratio.

The f-IGFBP3/t-IGFBP3 ratio serving as an index of IGFBP 3 fragmentation was influenced not only by age $(P<0.0001)$ but also by the diagnostic group $(P<0.006)$. There was no influence of an interaction between age and diagnostic group. Control children had higher ratios than CKD $(P=0.002)$ and DT $(P=0.0003)$ children. $C K D$ children $(P \leq 0.005)$ and
DT children $(P \leq 0.001)$ had lower ratios than children with GHD or SGA. RTx children had ratios higher than those of DT $(P=0.035)$ but lower than those of GHD $(P=0.016)$ children (data not shown).

\section{Association of IGFBP3 concentrations and GFR}

When controls and children with kidney diseases were analyzed together according to GFR, there was a significant negative correlation of GFR with t-IGFBP3 (Fig. 3, panel A) but not with f-IGFBP3 (Fig. 3, panel B).

\section{Group characteristics}

Healthy controls In healthy children serving as controls, the calculated GFR $(107.83 \pm 20.53 \mathrm{ml} / \mathrm{min}$ per $1.73 \mathrm{~m}^{2}$ ) was significantly higher than that of the patients with CKD, DT, and after RTx $(P<0.0001)$. The majority of the healthy control children had IGF1 SDS and t-IGFBP 3 SDS in the normal range throughout CA (mean $-0.68 \pm 1.07$ and $0.24 \pm 0.92$ respectively). In these children, both f-IGFBP 3 and t-IGFBP 3 increased with age $(r=0.80, P<0.0001$, and $r=0.49, P=0.007$; Fig. 1). f-IGFBP 3 was lowest at an age $<6$ years and increased to a mean concentration of $2.24 \pm 0.25 \mathrm{mg} / \mathrm{l}$ in
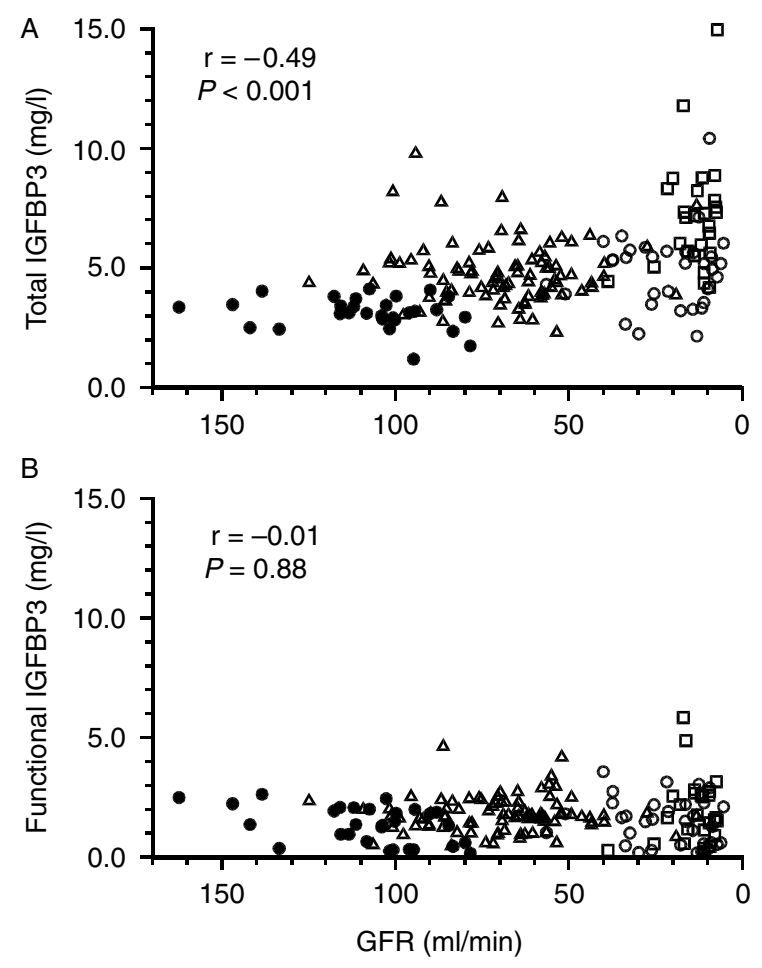

Figure 3 Correlation (Spearman rank) of IGFBP3 serum concentrations with glomerular filtration rate (GFR). (Panel A) Total (t)-IGFBP3. (Panel B) Functional (f)-IGFBP3. Closed circle, healthy children; open circle, children with chronic kidney disease stage 3-4; open square, children undergoing dialysis treatment; open triangle, children with post-renal transplantation. 
Table 2 Reference ranges of f-IGFBP3 and the f-/t-IGFBP3 ratio in healthy children and adolescents of different age all age groups or between pairs of groups labelled with.

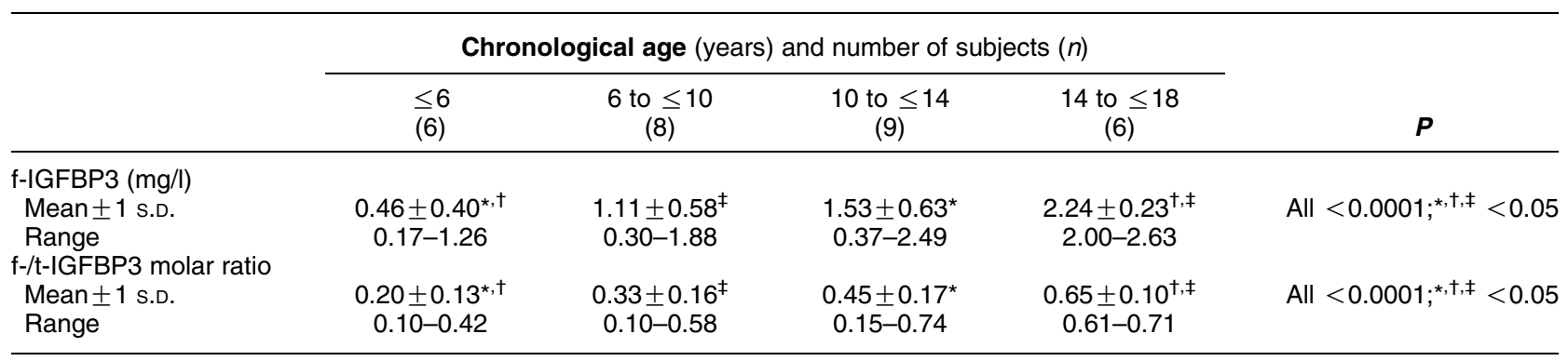

$P$ values (ANOVA) indicate differences between all age groups or between pairs of groups labelled with *, ${ }^{\dagger}$ or $^{\ddagger}$.

adolescents aged 14-18 years, and the f-/t-IGFBP 3 ratio increased from $0.20 \pm 0.13$ at ages $<6$ years to $0.65 \pm$ 0.10 in the 14 - to 18 -year olds (both $P<0.0001$ for all age groups) (Table 2 ). The f-/t-IGFBP 3 ratio significantly correlated with age $(r=0.77, P<0.0001)$ and GFR $(r=0.46, P=0.01)$. The IGF1/t-IGFBP3 molar ratio (mean $0.23 \pm 0.11$ s.D.) showed a positive correlation with CA $(r=0.74, P<0.0001)$ and a weak correlation with GFR $(r=0.39, P=0.035)$. No correlation was seen between CA (GFR) and the IGF1/f-IGFBP3 ratio, IGF1 SDS, and t-IGFBP3 SDS respectively.

CKD (CKD stage 3-4) The mean GFR in this group was $22.1 \pm 13.3 \mathrm{ml} / \mathrm{min}$ per $1.73 \mathrm{~m}^{2}$. IGF1 was low normal for $\mathrm{CA}$ (mean $-0.71 \pm 1.55$ s.D.). Throughout the age range, many t-IGFBP3 SDS were above normal (mean SDS $2.1 \pm 1.13$ s.D.). f-IGFBP3 (mean $1.48 \pm 0.96 \mathrm{mg} / \mathrm{l}$ ), t-IGFBP3 (mean $4.88 \pm 1.58 \mathrm{mg} / \mathrm{l}$ ), and the $\mathrm{f}-/ \mathrm{t}-\mathrm{IGFBP} 3$ molar ratio (mean $0.29 \pm 0.16$ s.D.) correlated with age $(r=0.57, P=0.0006 ; r=0.63, P<0.0001$; and $r=0.43, P=0.013$ respectively). Furthermore, there was a correlation between IGF1/tIGFBP3 (IGF1/f-IGFBP3) and CA with a mean $0.15 \pm 0.09$ s.D., $r=0.75$, and $P<0.0001$ (mean $0.59 \pm 0.29, r=0.48$, $P=0.004)$. No significant correlation was detected for the independent variables with GFR and for IGF1 SDS or t-IGFBP3 SDS with age.

DT group Mean GFR $\left(13.9 \pm 6.9 \mathrm{ml} / \mathrm{min}\right.$ per $\left.1.73 \mathrm{~m}^{2}\right)$ and the f-/t-IGFBP 3 ratio (mean $0.26 \pm 0.17$ s.D.) were lowest in this group. With a few exceptions, the data for IGF1 SDS were within the normal range according to CA (mean $0.35 \pm 1.70$ S.D.), but serum levels of t-IGFBP3 SDS were above normal in most children (mean $3.62 \pm 1.12$ s.D.) (Fig. 1). In this group, f-IGFBP3 (mean $1.91 \pm 1.36 \mathrm{mg} / \mathrm{l}, \quad r=0.53, P=0.005$ ) and t-IGFBP3 (mean $7.16 \pm 2.35 \mathrm{mg} / \mathrm{l}, r=0.45, P=0.02$ ) and the IGF1/t-IGFBP 3 ratio (mean $0.16 \pm 0.09$ s.D., $r=0.51, P=0.008)$ correlated with age. No significant correlation was seen between age and IGF1 SDS, t-IGFBP 3 SDS, the IGF $1 /$ f-IGFBP 3 ratio, or the f-IGFBP3/t-IGFBP3 ratio and between GFR and all dependent variables.
Renal transplantation The mean GFR (69.1 $\pm 19.6 \mathrm{ml} / \mathrm{min}$ per $1.73 \mathrm{~m}^{2}$ ) was increased over that of the CKD and DT groups but lower in the controls (all $P<0.0001$ ). Most IGF1 concentrations were normal (mean IGF1 SDS $0.33 \pm 1.28$ s.D.), but many t-IGFBP 3 concentrations were still elevated (mean t-IGFBP3 SDS $1.71 \pm 1.02$ s.D.) (Fig. 1). There was a negative correlation of both SDS with age $(r=-0.39$, $P=0.0002$ and $r=-0.24, P=0.02$ respectively) f-IGFBP3 (mean 1.78 $\pm 0.73 \mathrm{mg} / \mathrm{l}$ ) and t-IGFBP3 (mean $4.76 \pm 2.18 \mathrm{mg} / \mathrm{l})$ correlated with age $(r=0.51$, $P<0.0001$ and $r=0.26, P=0.01$ respectively). The mean IGF1/t-IGFBP3 ratio in this group of patients was $0.26 \pm 0.08$ s.D. and the mean IGF1/f-IGFBP3 ratio $0.76 \pm 0.29$ S.D. The mean value for the f-IGFBP3/t-IGFBP3 ratio was $0.38 \pm 0.15$ s.D. Positive correlations with age could be demonstrated for the IGF1/t-IGFBP3 ratio and f-IGFBP3/t-IGFBP3 ratio ( $r=0.47$ and $r=0.41$, both $P<0.0001)$. No significant correlation was seen between GFR and all dependent variables.

GH deficiency Due to the underlying disease, the IGF1 SDS was below the normal range (mean $-3.69 \pm 2.13$ S.D. ) in most children and correlated with age $(r=0.43$, $P=0.004)$; t-IGFBP3 SDS was decreased much less with a mean $-1.54 \pm 1.38$ s.D. (Fig. 1). f-IGFBP3 (mean $1.19 \pm 0.57 \mathrm{mg} / \mathrm{l}$ ), t-IGFBP3 (mean $2.61 \pm 0.82 \mathrm{mg} / \mathrm{l}$ ), IGF1/t-IGFBP3 ratio (mean $0.09 \pm 0.03$ s.D.), and the IGF1/f-IGFBP3 ratio (mean $0.20 \pm 0.10$ s.D.) correlated with age $(r=0.47, P=0.002 ; r=0.55, P=0.0002$; $r=0.61, P<0.0001 ; r=0.0 .47, P=0.0015$ respectively). The mean f-/t-IGFBP3 ratio was $0.45 \pm 0.13$ s.D. No significant correlation with age was seen for the f-/t-IGFBP3 ratio.

Small for gestational age In short children born SGA, IGF1 SDS was low for age (mean $-1.88 \pm 1.41$ S.D.) but t-IGFBP3 SDS was within the normal range for age (mean $-0.47 \pm 1.27$ s.D.) (Fig. 1). There was no correlation with age. f-IGFBP 3 and the IGF $1 /$ t-IGFBP 3 ratio showed mean values of $1.29 \pm 0.87 \mathrm{mg} / \mathrm{l}$ and $0.11 \pm 0.03$ s.D., respectively, and correlated with age 
( $r=0.37, P=0.032$, and $r=0.38, P=0.028)$. t-IGFBP3 (mean $3.30 \pm 1.06 \mathrm{mg} / \mathrm{l}$ ), IGF1/f-IGFBP3 ratio (mean $0.31 \pm 0.13$ s.D.), and the f-/t-IGFBP3 ratio (mean $0.38 \pm 0.17$ S.D.) in this group of patients did not correlate with age.

\section{Discussion}

IGF1 bioavailability is regulated by binding to IGFBP3; the release of IGF1 from its binding proteins depends on IGFBP3 fragmentation. During uremia, the IGF/IGFBP axis is altered with an increased proteolytic cleavage of IGFBP3 (29). Measurement of t-IGFBP3 with most commercial assays detects not only active moieties capable of IGF1 binding but also inactive fragments, whose proportion depends on the stage of renal insufficiency. Therefore, t-IGFBP3 is not a reliable parameter for the assessment of active IGFBP 3 under this condition.

In search of alternatives for the assessment of alterations of the $\mathrm{GH}$ axis in CKD patients, we prospectively measured f-IGFBP 3 in a cohort of pediatric patients with different stages of CKD, end-stage renal disease under DT, and after RTx. We compared these data with healthy controls and patients with GHD and SGA.

Our data show highly elevated t-IGFBP3 in patients with different stages of CKD, with a strong negative correlation of this parameter with GFR. t-IGFBP3 declines after RTx, but as GFR may still be lower than in healthy children, t-IGFBP3 levels have not returned to normal in more than a third of transplanted patients. Some but not all children with GHD show low levels of t-IGFBP 3 and low t-IGFBP3 SDS, consistent with the pathogenesis of their disease.

In contrast, f-IGFBP3 did not differ between healthy children and patients with CKD, GHD, and SGA. A correlation with GFR was not seen in the CKD group. These findings support the hypothesis that in CKD, available IGFBP 3 assays measure predominantly inactive IGFBP3 fragments. Inactive fragments may also cause the f-IGFBP $3 /$ t-IGFBP 3 ratio to be significantly lower in CKD and DT children compared with healthy children and children with GHD and SGA. RTx children had higher f-/t-IGFBP3 ratios than DT children.

In all diagnostic groups, f-IGFBP3 increases with age, correlations showing $r$ values from 0.37 to 0.8 . Except for the SGA children, positive correlations with age were also found for t-IGFBP3.

This would indicate that the influence of age on both $\mathrm{f}$ - and t-IGFBP3 present in healthy children is modified but not abolished by impaired renal function. Interestingly, in young healthy children, the f-/t-IGFBP3 molar ratio is low. In children below the age of 6 years, up to $80 \%$ of the t-IGFBP 3 immunoreactivity consists of fragments. The f-/t-IGFBP3 molar ratio then increases significantly with age to adolescent values that are similar to those described for a group of healthy adult blood donors (21). In our patients and controls, the rise observed in f-IGFBP3 throughout childhood and adolescence is higher than that of t-IGFBP3. This is in line with Gargosky et al. (30) who, studying the proportion of fragmented IGFBP 3 from early childhood to adolescence, found the ratio of fragmented IGFBP3 over the sum of all IGFBP 3 variants in urine to be higher in young children and to decrease with age. The physiological relevance of this higher fragmentation at young age is yet unclear. These observations could be seen to indicate that the rate of IGFBP3 proteolysis decreases or the elimination of fragments from the circulation increases with age. Limitations apply concerning our reference group with regard to the small patient number, not allowing for stratification according to pubertal stages, or for separate analysis according to sex in pubertal subjects, as a sex difference for f-IGFBP3 has been found in adults (21).

Routinely, IGF 1 and t-IGFBP 3 are measured in patients with CKD to monitor the GH/IGF axis (20). Alterations of these parameters in combination with decrease in height velocity lead to initiation and adjustment of GH therapy. Depending on our results, t-IGFBP 3 measurements alone are not sufficient to assess the impairment of the somatotropic axis as they do not reflect the degree of IGFBP3 fragmentation. More specific measuring techniques include isotope dilution mass spectrometry, a method that is very complex, labor intensive, and sensitive to interfering factors (31). To assess fragmentation, direct measurement of the functional component f-IGFBP3 by immunoassay, and calculating its ratio to t-IGFBP3, is a practicable alternative. However, the overall significance of f-IGFBP3 for assessing the state of the somatotropic axis appears to be low, as f-IGFBP3 did not differ between healthy children, children with CKD, and children with GHD and SGA. In addition, especially older patients with GHD had normal serum concentrations of both parameters.

In CKD, IGF1 SDS was within the normal range but slightly decreased compared with healthy controls, whereas dialysis patients as well as patients following RTx with their minor impairment of renal function displayed IGF1 SDS levels higher than healthy controls. This is consistent with the literature, where immunoactive IGF1 levels are described to be normal or even elevated despite reduced production and bioactivity $(15,16,32)$. This observation was explained by the prolonged half-life due to IGFBP 3 binding in the presence of high t-IGFBP3 levels.

Our finding that f-IGFBP3 measuring exclusively IGFBP 3 variants capable of IGF1 binding does not differ from levels of healthy controls indicates that mechanisms other than binding to intact IGFBP3 may be operative in IGF inactivation caused by renal failure.

\section{Conclusions}

Our data indicate that in CKD, DT, and RTx patients, the elevation of t-IGFBP3, as measured by 
conventional assays, is probably due to the accumulation of non-IGF1-binding, inactive IGFBP fragments. The low functional to total ratio in these groups is compatible with this notion. Simultaneous measurement of $\mathrm{f}$ - and t-IGFBP 3 could provide an index of IGFBP 3 fragmentation. The fact that f-IGFBP 3 concentrations in CKD, DT, and RTx are not different from controls would challenge the concept of growth failure caused by excessive IGFBP 3 concentrations blocking IGF1 action under these conditions.

\section{Declaration of interest}

A K Büscher, R Büscher, P F Hoyer, and B P Hauffa declared no competing interests. L Pridzun, R Langkamp, and $\mathrm{N}$ Wachendorfer are employees of Mediagnost $\mathrm{GmbH}$, the company producing the functional IGFBP3 ELISA.

\section{Funding}

This research did not receive any specific grant from any funding agency in the public, commercial, or not-for-profit sector.

\section{Acknowledgements}

The authors thank Nils Lehmann, Institute for Medical Informatics, Biometry and Epidemiology, University of Duisburg-Essen for helpful comments regarding the statistical analyses.

\section{References}

1 Seikaly MG, Ho PL, Emmett L, Fine RN \& Tejani A. Chronic renal insufficiency in children: the 2001 Annual Report of the NAPRTCS. Pediatric Nephrology 200318 796-804. (doi:10. 1007/s00467-003-1158-5)

2 Seikaly MG, Salhab N, Gipson D, Yiu V \& Stablein D. Stature in children with chronic kidney disease: analysis of NAPRTCS database. Pediatric Nephrology 200621 793-799. (doi:10.1007/ s00467-006-0040-7)

3 Mehls O, Wuhl E, Tönshoff B, Schaefer F, Nissel R \& Haffner D. Growth hormone treatment in short children with chronic kidney disease. Acta Paediatrica 200897 1159-1164. (doi:10.1111/ j.1651-2227.2008.00845.x)

4 Roelfsema V \& Clark RG. The growth hormone and insulin-like growth factor axis: its manipulation for the benefit of growth disorders in renal failure. Journal of the American Society of Nephrology 200112 1297-1306.

5 Seikaly MG, Salhab N, Warady BA \& Stablein D. Use of rhGH in children with chronic kidney disease: lessons from NAPRTCS. Pediatric Nephrology 200722 1195-1204. (doi:10.1007/s00467007-0497-z)

6 Seikaly MG, Waber P, Warady BA \& Stablein D. The effect of rhGH on height velocity and BMI in children with CKD: a report of the NAPRTCS registry. Pediatric Nephrology 200924 1711-1717. (doi:10.1007/s00467-009-1183-0)

7 Nissel R, Brazda I, Feneberg R, Wigger M, Greiner C, Querfeld U \& Haffner D. Effect of renal transplantation in childhood on longitudinal growth and adult height. Kidney International 2004 66 792-800. (doi:10.1111/j.1523-1755.2004.00805.x)

8 Frystyk J, Ivarsen P, Skjærbæk C, Flyvbjerg A, Pedersen EB \& Orskov H. Serum-free insulin-like growth factor I correlates with clearance in patients with chronic renal failure. Kidney International 199956 2076-2084. (doi:10.1046/j.1523-1755.1999. 00798.x)
9 Baxter RC. Insulin-like growth factor (IGF)-binding proteins: interactions with IGFs and intrinsic bioactivities. American Journal of Physiology. Endocrinology and Metabolism $2000 \mathbf{2 7 8}$ E967-E976.

10 Mohan S \& Baylink DJ. IGF-binding proteins are multifunctional and act via IGF-dependent and -independent mechanisms. Journal of Endocrinology 2002175 19-31. (doi:10.1677/joe.0. 1750019)

11 Juul A, Flyvbjerg A, Frystyk J, Müller J \& Skakkebaek NE. Serum concentrations of free and total insulin-like growth factor-I, IGF binding proteins- 1 and -3 and IGFBP- 3 protease activity in boys with normal or precocious puberty. Clinical Endocrinology 199644 515-523. (doi:10.1046/j.1365-2265.1996.711531.x)

12 Jones JI \& Clemmons DR. Insulin-like growth factors and their binding proteins: biological actions. Endocrine Reviews 199516 3-34. (doi:10.1210/edrv-16-1-3)

13 Lamson G, Giudice LC, Cohen P, Liu F, Gargosky S, Muller HL, Oh Y, Wilson KF, Hintz RL \& Rosenfeld RG. Proteolysis of IGFBP-3 may be a common regulatory mechanism of IGF action in vivo. Growth Regulation 19933 91-95.

14 Tönshoff B, Kiepe D \& Ciarmatori S. Growth hormone/insulin-like growth factor system in children with chronic renal failure. Pediatric Nephrology 200520 279-289. (doi:10.1007/s00467005-1821-0)

15 Tönshoff B, Blum WF, Wingen AM, Mehls O, Rätsch I, Michelis K, Kapogiannis T, Lennert T, Jung F, Gellert S, Tulassay T, Sallay P, Von Lilien T, Querfeld U, Von Wendt-Gökner MA, Bonzel KE, Gusmano R, Verrina E, Offner G, Fabian-Bach C, Appiani A, Bettinelli A, Feber J \& Picca S. Serum insulin-like growth factors (IGFs) and IGF binding proteins 1, 2, and 3 in children with chronic renal failure: relationship to height and glomerular filtration rate. Journal of Clinical Endocrinology and Metabolism 199580 2684-2691. (doi:10.1210/jc.80.9.2684)

16 Houang M, Cabrol S, Perin L, Ducos B, Bensman A \& Le Bouc Y. Insulin-like growth factor-I (IGF-I), insulin-like growth factor binding proteins (IGFBP) and insulin-like growth factor type I receptor in children with various status of chronic renal failure. Growth Hormone \& IGF Research 200010 332-341. (doi:10. 1054/ghir.2000.0178)

17 Tönshoff B, Blum WF \& Mehls O. Derangements of the somatotropic hormone axis in chronic renal failure. Kidney International. Supplement 199758 S106-S113.

18 Blum WF, Ranke MB, Kietzmann K, Tönshoff B \& Mehls O. Growth hormone resistance and inhibition of somatomedin activity by excess of insulin-like growth factor binding protein in uraemia. Pediatric Nephrology 19915 539-544. (doi:10.1007/ BF01453697)

19 Ding H, Gao XL, Hirschberg R, Vadgama JV \& Kopple JD. Impaired actions of insulin-like growth factor 1 on protein synthesis and degradation in skeletal muscle of rats with chronic renal failure. Evidence for a postreceptor defect. Journal of Clinical Investigation 199697 1064-1075. (doi:10.1172/JCI118499)

20 Rees L \& Kovacs G. Growth and puberty. In ESPN Handbook, pp 383-384. Eds Cochat P. Lyon: Medcom, 2002.

21 Langkamp M, Weber K, Kirschner M, Pridzun L \& Ranke MB. Validation of functional insulin-like growth factor binding protein3 measurement by a ligand immunoassay. Clinical Laboratory $201056535-542$.

22 National Kidney Foundation. Part 4. Definition and classification of stages of chronic kidney disease. Guideline 1. Definition and stages of chronic kidney disease. American Journal of Kidney Diseases 200239 S46-S75. (doi:10.1053/ajkd.2002.30943)

23 Blum WF \& Breier BH. Radioimmunoassays for IGFs and IGFBPs. Growth Regulation 19944 (Suppl 1) 11-19.

24 Giovannucci E. Nutrition, insulin, insulin-like growth factors and cancer. Hormone and Metabolic Research 200335 694-704. (doi:10.1055/s-2004-814147)

25 Schwartz GJ, Brion LP \& Spitzer A. The use of plasma creatinine concentration for estimating glomerular filtration rate in infants, children, and adolescents. Pediatric Clinics of North America 1987 34 571-590. 
26 McCusker RH, Busby WH, Dehoff MH, Camacho-Hubner C \& Clemmons DR. Insulin-like growth factor (IGF) binding to cell monolayers is directly modulated by the addition of IGF-binding proteins. Endocrinology 1991129 939-949. (doi:10.1210/endo129-2-939)

27 Firth SM \& Baxter RC. Cellular actions of the insulin-like growth factor binding proteins. Endocrine Reviews 200223 824-854. (doi:10.1210/er.2001-0033)

28 De Mellow JS \& Baxter RC. Growth hormone-dependent insulinlike growth factor (IGF) binding protein both inhibits and potentiates IGF-I-stimulated DNA synthesis in human skin fibroblasts. Biochemical and Biophysical Research Communications 1988156 199-204. (doi:10.1016/S0006-291X(88)80824-6)

29 Durham SK, Mohan S, Liu F, Baker BK, Lee PD, Hintz RL, Conover CA \& Powell DR. Bioactivity of a 29-kilodalton insulinlike growth factor binding protein-3 fragment present in excess in chronic renal failure serum. Pediatric Research 199742 335-341. (doi:10.1203/00006450-199709000-00014)

30 Gargosky SE, Hasegawa T, Tapanainen P, MacGillivray M, Hasegawa Y \& Rosenfeld RG. Urinary insulin-like growth factors
(IGF) and IGF-binding proteins in normal subjects, growth hormone deficiency, and renal disease. Journal of Clinical Endocrinology and Metabolism $1993 \mathbf{7 6}$ 1631-1637. (doi:10. 1210/jc.76.6.1631)

31 Kirsch S, Widart J, Louette J, Focant JF \& De Pauw E. Development of an absolute quantification method targeting growth hormone biomarkers using liquid chromatography coupled to isotope dilution mass spectrometry. Journal of Chromatography A 2007 1153 300-306. (doi:10.1016/j.chroma.2007.03.058)

32 Blum WF. Insulin-like growth factors (IGFs) and IGF binding proteins in chronic renal failure: evidence for reduced secretion of IGFs. Acta Paediatrica Scandinavica 199180 (Suppl 379) 24-31. (doi:10.1111/j.1651-2227.1991.tb12039.x)

Received 26 October 2011

Revised version received 26 January 2012

Accepted 8 February 2012 